\title{
Gradhiva
}

\section{Edna G. Bay Asen, Ancestors and Vodun. Tracing Change in African Art}

Urbana et Chicago, University of Illinois Press, 2008, 186 p.

\section{Emmanuelle Kadya Tall}

\section{OpenEdition}

\section{Journals}

Édition électronique

URL : http://journals.openedition.org/gradhiva/1246

DOI : $10.4000 /$ gradhiva. 1246

ISSN : 1760-849X

\section{Éditeur}

Musée du quai Branly Jacques Chirac

Édition imprimée

Date de publication : 15 novembre 2008

Pagination : 153-154

ISBN : 978-2-915133-94-3

ISSN : 0764-8928

\section{Référence électronique}

Emmanuelle Kadya Tall, «Edna G. Bay Asen, Ancestors and Vodun. Tracing Change in African Art »,

Gradhiva [En ligne], 8 | 2008, mis en ligne le 10 décembre 2008, consulté le 21 septembre 2020. URL http://journals.openedition.org/gradhiva/1246 ; DOI : https://doi.org/10.4000/gradhiva.1246

Ce document a été généré automatiquement le 21 septembre 2020.

(c) musée du quai Branly 


\section{Edna G. Bay Asen, Ancestors and Vodun. Tracing Change in African Art}

Urbana et Chicago, University of Illinois Press, 2008, 186 p.

Emmanuelle Kadya Tall

\section{RÉFÉRENCE}

Edna G. Bay Asen, Ancestors and Vodun. Tracing Change in African Art, Urbana et Chicago, University of Illinois Press, 2008, 186 p.

1 Dès son titre, le dernier ouvrage d'Edna Bay évoque les travaux de Ranger et Hosbawn sur l'invention des traditions, et nous sommes déjà avertis de l'ampleur de la tâche que l'auteur du magnifique livre Wifes of the Leopard ${ }^{1} \mathrm{~s}^{\prime} e s t$ fixé: traquer les changements dans un art qui conjugue une pratique artisanale nouvelle à un rituel plus ancien de mise en relation des vivants avec le monde des morts. Ce monde des morts est lui-même un monde intermédiaire entre le monde terrestre et le monde supranaturel des vodun, ces entités sacrées qui régissent les mystères du monde dans sa totalité. En effet, évoquer les morts, c'est aussi évoquer le monde des vodun puisque certains morts, du fait de leur destin exceptionnel, atteignent le statut de vodun. La royauté aboméenne, voyant sa puissance décliner au milieu du xix ${ }^{\mathrm{e}}$ siècle, stimule l'invention de nouveaux cultes dynastiques, celui des Nesuxwe - enfants monstres à l'origine des différentes dynasties aboméennes -, et de nouveaux autels - asen - qui permettent de glorifier en images les anciens rois. Mais au-delà de l'imagerie nouvelle que constitue l'érection de tiges métalliques terminées en leur faîte par une coupelle surmontée des emblèmes ou des faits d'armes, déclinés sous forme de rébus, des anciens rois d'Abomey, ces asen sont de véritables objets-dieux qu'on alimente à périodicité régulière, car d'eux émanent, à l'instar des vodun, une puissance dont bénéficient les vivants qui les honorent.

2 Le contexte sociohistorique du XIXe siècle favorise ces inventions dans la mesure où le contexte international n'est plus propice à la traite, et si le roi Guezo avait déjà commencé à investir dans l'économie de plantation, celle-ci ne sera jamais aussi 
lucrative que la traite des esclaves. Il semble donc qu'il faille donner à voir, de manière empirique, que le royaume d'Abomey est encore puissant, et c'est à cette tâche que son successeur, le roi Glélé, semble s'être employé durant son règne.

3 L'ouvrage se décompose en sept chapitres qui se déroulent de manière chronologique, et si le premier chapitre, inscrivant le culte des ancêtres dans un contexte anthropologique et linguistique est un peu ardu, les suivants sont plutôt fluides et bien illustrés - même si parfois le lecteur peut demeurer frustré de ne pas avoir une vision d'ensemble des cultes aux ancêtres dans cette région. Il est vrai que le projet de croiser trois disciplines (histoire, anthropologie et histoire de l'art) est ambitieux, et l'on comprend sans mal que l'auteur ait mis plus de vingt ans à faire aboutir son projet. Il est certain que la tâche de faire l'histoire de la région est toujours malaisée, en l'absence de documents écrits au-delà de la période des premiers contacts avec les Européens, et nul doute que c'est à l'archéologie que reviendra le rôle de nous éclairer définitivement, notamment sur le rôle de la métallurgie dans cette région. En effet, en lisant la partie de l'ouvrage consacrée aux forgerons-bijoutiers du royaume, notamment la famille Hontoundji (chap. III), on en vient à se demander si ces artisans, qui ont su préserver jalousement certaines techniques empruntées à d'autres, ne sont pas des «imposteurs» venus d'ailleurs, sans lien avec la métallurgie ancienne, que les fouilles récentes dans la région d'Abomey, ou encore du Mono, ont fait réapparaître. Nous avons, en effet, toujours été étonnée de voir que, dans un royaume guerrier comme celui d'Abomey, le forgeron-bijoutier occupait certes une place de choix dans la mesure où il intégrait les lignages dynastiques, mais que cette place semblait obéir plus à une logique clientéliste - que Houseman et al ${ }^{2}$ ont bien analysée - qu'à la fonction qu'il pouvait occuper dans un univers guerrier.

4 Par ailleurs, si Edna Bay rend convaincante sa démonstration de l'émergence des autels asen et leur popularisation au sein de la population Fon, on regrettera qu'elle évoque à peine les autres manières qu'ont les populations voisines et/ou colonisées des Fon d'Abomey d'honorer les ancêtres. Si, en effet, le culte kutito est un emprunt au culte des egungun des Yoruba, l'expérience de terrain nous a appris que la manière la plus sûre de déterminer à quelle appartenance un groupe s'identifiait dans cette région balayée par de nombreuses vagues migratoires était justement d'en observer les rites funéraires. Or, à notre connaissance, il est difficile de voir un descendant d'Aguda ou d'un Peda à Ouidah honorer ses ancêtres à travers des asen. Il nous semble donc que si certaines techniques ou certains média (comme la photographie ou la vidéo) sont indifféremment utilisés par l'ensemble des populations du Sud-Bénin, ou si des pratiques rituelles «identitaires» sont détournées par d'autres (oro, egungun, par exemple), ce détournement aurait mérité un chapitre. En effet, il est intéressant de noter que c'est justement dans la sphère des cultes aux ancêtres qu'aujourd'hui se manifeste un regain d'intérêt de certains segments de la population pour des rituels dont la dimension identitaire est abandonnée au profit de la dimension «magique».

On notera cependant, tout au long des chapitres, la rigueur de l'analyse dans la mise en écho d'entretiens menés par l'auteur à deux périodes différentes de son activité de terrain, ainsi que la mise en perspective de ses matériaux avec des interprétations recueillies par des observateurs antérieurs. Leur versatilité témoigne de la nécessité de toujours les resituer dans le contexte dans lequel elles ont été émises. On retiendra avec les interlocuteurs d'Edna Bay que l'auteur d'une œuvre et son commanditaire sont seuls 
capables d'en déchiffrer le sens voulu, tout en rappelant néanmoins qu'il appartient à chacun de se l'approprier en l'interprétant à sa manière.

Précisons par ailleurs que nous avons particulièrement apprécié le chapitre consacré à l'impact de la colonisation dans la popularisation des autels asen comme biens de prestige. Edna Bay y analyse de manière extrêmement pertinente le rôle des administrateurs coloniaux et des missionnaires dans l'élaboration d'une ethnographie qui va servir de repère aux élites naissantes. Ainsi, dans le prolongement de son analyse, on pourrait dire que cette entreprise coloniale assimilationniste férue de «traditions africaines» est à la base d'un imaginaire national avec lequel Mathieu Kérékou a voulu rompre en transformant l'ancienne colonie du Dahomey en Bénin au milieu des années 1970. Il nous semble, en effet, que l'incorporation des attributs royaux aboméens dans la mode vestimentaire des élites naissantes participe de cet imaginaire hérité de la colonisation qui démembra le royaume d'Abomey. Cet imaginaire sera conservé, figé dans un temps immobile, celui de la tradition, où les marques de distinction devaient être empruntées au pouvoir aboméen, à ses hiérarchies organisées de la manière la plus sophistiquée, au moment même du déclin du royaume.

7 Au-delà des quelques réserves suscitées par la lecture de cet ouvrage, retenons qu'il foisonne de questionnements, d'intuitions qui permettent de réfléchir avec profit sur l'actualité et l'activité rituelle intense dont le Bénin méridional est familier.

\section{NOTES}

1. Wives of the Leopard: Gender, Politics and Culture in the Kingdom of Dahomey. Charlottesville, University Press of Virginia, 1998.

2. Michael Houseman et al. « Notes sur la structure évolutive d'une ville historique. L'exemple d'Abomey », Cahiers d'études africaines 104, xxxvi-4, 1986 : 527-546.

\section{AUTEURS}

\section{EMMANUELLE KADYA TALL}

Kadya.Tall@ehess.fr 